\title{
Introduction - Les opérations marchandes. Pourquoi et comment des transactions marchandes ont-elles lieu?
}

\section{Geneviève Teil}

\section{OpenEdition}

Journals

\section{Édition électronique}

URL : http://journals.openedition.org/economierurale/3151

DOI : 10.4000/economierurale.3151

ISSN : 2105-2581

Éditeur

Société Française d'Économie Rurale (SFER)

Édition imprimée

Date de publication : 5 juillet 2005

Pagination : 05-07

ISSN : 0013-0559

\section{Référence électronique}

Geneviève Teil, «Introduction - Les opérations marchandes. Pourquoi et comment des transactions marchandes ont-elles lieu ? », Économie rurale [En ligne], 286-287 | Mars-juin 2005, mis en ligne le 05 mai 2007, consulté le 19 avril 2019. URL : http://journals.openedition.org/economierurale/3151; DOI : 10.4000/economierurale.3151

Ce document a été généré automatiquement le 19 avril 2019.

(c) Tous droits réservés 


\title{
Introduction - Les opérations marchandes. Pourquoi et comment des transactions marchandes ont- elles lieu?
}

\author{
Geneviève Teil
}

\section{NOTE DE L'AUTEUR}

Les articles de ce dossier sont issus d'un travail collectif mené pendant plus de trois ans au sein du groupe de travail « Mise En Marché » qui a rassemblé de nombreux chercheurs en marketing, économie, anthropologie, ethnologie, sociologie provenant de laboratoires divers, CNRS, EHESS, Ecole Normale Supérieure, Ecole des Mines et autres écoles d'ingénieurs, France Telecom, INRA, universités françaises, suisses, belges ou luxembourgeoises. Nous remercions les auteurs du numéro bien sûr, mais aussi tous les participants du séminaire dont les contributions, discussions, présentations d'ouvrages ont accompagné et enrichi ce numéro.

1 Cette question a nourri de très nombreux travaux empiriques sur les relations entre les entreprises; elle est restée plus discrète à propos des consommateurs, de leurs achats, où les réponses tiennent en quelques hypothèses : structuration sociale des "préférences » des consommateurs; «manipulation» par les vendeurs ou les techniques marchandes; connaissance de la «demande » et ajustement de «l'offre » grâce aux techniques du marketing. Toutes ces théories confinent l'acteur consommateur dans un rôle passif que l'on a vu récemment se fracturer. Le consommateur (appelé consommacteur dans certaines entreprises) ne tient pas ou ne tient plus dans la place qui lui a été réservée. La première structuration en « classes », « segments » ou "styles » de ce collectif instable d'acheteurs a débouché sur de nouvelles théories dans lesquelles le consommateur était tenu pour plus individualiste et réflexif. Cependant, cette réflexivité qui s'articulait difficilement 
avec les anciennes dispositions préétablies a obligé à repenser les interactions marchandes et la «félicité » de la rencontre produit consommateur, pour reprendre l'expression de E. Goffman.

Ce consommateur réflexif a conduit à suspendre les théories de la consommation qui attribuaient aux consommateurs des goûts, des intérêts, des attentes, des désirs, d'origine physiologique, psychologique ou sociale, et grâce auxquels on expliquait l'émergence et le développement des marchés : "New medical markets developed in response to the needs of a growing population.» (Mann Wall, 2003) ${ }^{1}$ En effet, ces "besoins", "attentes", "préférences » naturalisés dans la personne du consommateur ne permettaient guère que des explications très rétrospectives de l'évolution des marchés. Une autre solution a émergé qui les interprète comme le résultat de l'action des intermédiaires de marché. Il s'agit ici d'expliciter la manière dont les intermédiaires tentent de saisir le collectif abstrait de leurs consommateurs afin d'organiser et orienter leur rencontre avec les produits. D'autres études ont, au contraire, exploré les dispositifs de rencontre entre le consommateur et les produits et pointé notamment une caractéristique nouvelle des produits de consommation, leur variété. L'ouvrage historique de R. L. Blaszcszyk (2000) ${ }^{2}$ montre ainsi de manière particulièrement détaillée la modification des interactions entre les consommateurs et les produits suite à l'industrialisation de quelques procédés de production d'objets de consommation devenus ordinaires. Il fait ressortir en particulier le rôle du choix nouvellement offert aux clients qui leur permet d'avoir des préférences, et, de fil en aiguille, de se doter de goûts puis d'en changer. Ainsi la notion de "demande " s'est-elle effritée laissant place à des procédures de saisie et d'accumulation ou d'agrégation d'acteurs.

3 Cette déconstruction de la notion de demande s'est accompagnée d'une déconstruction parallèle de celle de l'offre à la suite notamment de nombreux travaux sur la qualification des produits. La différenciation qualitative et les controverses qu'elles engendraient on en effet conduit à revoir une hypothèse souvent implicite selon laquelle les qualités des choses se donnaient à saisir et appréhender de manière triviale : un chou est un chou, un clou, un clou et un chat, un chat. Ce sont d'abord les théories de l'asymétrie de l'information qui ont mis à mal l'existence d'un savoir collectif partagé sur la nature des marchandises, en pointant le fait que les clients pouvaient être particulièrement démunis au moment de l'évaluation de la qualité des objets ou services attendus, sans que cela condamne l'existence d'échanges marchands (voir Karpik, 1995)³. Cependant, des études de cas ont indiqué que ces connaissances sur la qualité des produits n'étaient pas toujours partagées par des producteurs, elles pouvaient même les diviser. Elles résultaient surtout de procédures d'investigation des propriétés des objets. Ces théories de l'asymétrie de l'information supposaient aussi que les techniques de connaissance des producteurs leur conféraient un point de vue plus "vrai » et supérieur à celui du consommateur, indépendamment de la saisie propre que le consommateur pouvait en avoir ou que lui conférait son propre usage. Or si les producteurs en savaient plus sur le procédé de fabrication, les consommateurs en savaient plus sur la destination des produits. Et - le marketing a longuement insisté là dessus - la déraison, le manque d'information ou l'irrationalité que les producteurs pouvaient attribuer à leurs clients pouvait aussi être interprétée comme une méconnaissance des clients et de leurs usages des produits de la part des producteurs.

Les deux piliers qui soutenaient les interprétations de la félicité des accords marchands se sont donc délités entraînant avec eux les interprétations naturelles de la rencontre client 
produit par correspondance entre les caractéristiques des uns et les dispositions des autres, mais aussi les théories de la domination ou de la manipulation des acheteurs par les vendeurs. Le vide qu'ils laissent est cependant loin d'être comblé malgré d'importants travaux (Callon, 1998)

Ce numéro tente de poursuivre l'effort initié et cherche à contribuer à une meilleure description des processus qui accompagnent les transactions marchandes, en particulier celles qui concernent les consommateurs.

Plusieurs auteurs ont décrit les procédures par lesquelles les entreprises tentent de constituer et saisir le collectif de leurs clients puis de le stabiliser. En introduction de ce thème, Geneviève Teil décrit une alternative à la figuration du client et la configuration de la «demande ». La connaissance du consommateur, sa saisie, sa stabilisation ou son suivi, qui relèvent de techniques du marketing dit «de la demande » et cherchent à toujours mieux saisir le consommateur pour lui adapter un produit ne sont pas les seules manières de procéder. La préparation de la vente n'est pas toujours organisée comme une adaptation à un collectif configuré en "demande ", mais peut au contraire résulter de la configuration d'une « offre » qui laisse le consommateur très libre quant à la production de ses propres attachements au produit.

Du côté du produit, deux articles décrivent deux cas de différenciation de produits. La qualité fermière de la viande de bœuf n'aurait pu exister sans les controverses sur le bœuf aux hormones qui a fait émerger une attention envers le caractère artificiel ou naturel de l'alimentation et permis de fissurer un ensemble de savoir-faire fortement imbriqués et solidifiés dans des procédures, des instruments... Le premier article, de Pierre Stassart, sur le marché fermier de la viande bovine belge, montre à quel point la qualification d'un produit reste toujours une procédure de représentation fragile, jamais définitivement stabilisée. Le second article, de Thomas Debril, sur la commercialisation des huîtres propose une situation assez opposée. Il montre le travail de qualification dont les huitres ont récemment fait l'objet et qui a fait naître simultanément deux modalités de saisie de la consommation. La grande distribution s'est appuyée sur une représentation culturelle de la consommation d'huître, produit festif consommée à une époque précise de l'année, pour organiser et optimiser les flux de marchandise. Au contraire, les producteurs, qui se sentaient trop liés par cette organisation, ont tenté d'y échapper en organisant la formation d'une rencontre alternative «non culturelle » des huîtres, une consommation d'amateurs éclairés, informés, curieux, intéressés par les qualités des produits.

8 Les trois derniers articles abordent une question encore assez peu traitée dans la littérature, si ce n'est dans des cas bien particuliers (Zelizer, 1994)5, celui de l'appréciation. Celui d'Horacio Ortiz s'attache à décrire la multiplicité des procédures d'évaluation qui imprègnent l'activité des brokers, analystes et gérants sur les marchés financiers. Ces procédures passent continûment de l'évaluation des actions à l'évaluation des outils d'évaluation, des évaluateurs et à leur rémunération. Ce cas montre que l'appréciation repose sur des procédures précises, multiples, discordantes qui nécessitent à la fois des choix et des arbitrages. Enfin, Christian Licoppe reprend cette question dans le cas de l'achat de produits de tourisme et voyage qui confronte l'acheteur à une différenciation non pas « qualitative » des biens, mais « appréciative ». Ces acheteurs ont en effet le choix entre deux dispositifs de formation de prix : des enchères ou des prix fixes sur catalogue. L'étude des interactions entre les acheteurs et les vendeurs fait ressortir l'habileté économique des uns et des autres mais aussi les interprétations et les soupçons qui structurent les échanges marchands. 


\section{NOTES}

1. . Mann Wall B. Healthcare as product - Catholic Sisters confront charity and the hospital marketplace, 1865-1925. In S. Strasser Ed(s), "Commodifying Everything - Relationships of the Market”, Routledge, New York \& Lndn, 2003, 145, p. 143-168.

2. . Blaszcszyk R. L. Imagining consumers : design and innovation from Wedgwood to Corning. John Hopkins University Press, Baltimore \& London, 2000.

3. Karpik L. Les avocats entre l'État, le public et le marché, XIIIe-XXe siècle. Gallimard, Paris, 1995.

4. . Callon M. (Eds). The laws of the markets. Blackwell, Oxford, 1998.

5. . Zelizer V. A. Pricing the Priceless Child: The Changing Social Value of Children. Princeton Univerty Press, Princeton, 1994.

\section{AUTEUR}

\section{GENEVIÈVE TEIL}

Institut national de la recherche agronomique, SADAPT, Coordonnateur du dossier 